\title{
Article \\ Fractional-Order Discrete-Time SIR Epidemic Model with Vaccination: Chaos and Complexity
}

\author{
Zai-Yin $\mathrm{He}^{1}{ }^{\text {, Abderrahmane Abbes }}{ }^{2}$ (D), Hadi Jahanshahi ${ }^{3}$, Naif D. Alotaibi ${ }^{4}$ and Ye Wang ${ }^{5,6, *(\mathbb{D})}$ \\ 1 School of Mathematics, Hunan University, Changsha 410082, China; hzy@zjhu.edu.cn \\ 2 Department of Mathematics, University of Jordan, Amman 11942, Jordan; abder.abbes@gmail.com \\ 3 Department of Mechanical Engineering, University of Manitoba, Winnipeg, MB R3T 5V6, Canada; \\ jahanshahi.hadi90@gmail.com \\ 4 Department of Electrical and Computer Engineering, Faculty of Engineering, King Abdulaziz University, \\ Jeddah 21589, Saudi Arabia; ndalotabi@kau.edu.sa \\ 5 Department of Mathematics, Huzhou University, Huzhou 313000, China \\ 6 Institute for Advanced Study Honoring Chen Jian Gong, Hangzhou Normal University, \\ Hangzhou 311131, China \\ * Correspondence: 03019@zjhu.edu.cn
}

check for

updates

Citation: He, Z.Y.; Abbes, A.;

Jahanshahi, H.; Alotaibi, N.D.; Wang, Y. Fractional-Order Discrete-Time SIR Epidemic Model with Vaccination: Chaos and Complexity. Mathematics 2022, 10, 165. https://doi.org/ $10.3390 /$ math10020165

Academic Editors: Alexandra M.S.F. Galhano, António M. Lopes and Carlo Cattani

Received: 17 December 2021

Accepted: 2 January 2022

Published: 6 January 2022

Publisher's Note: MDPI stays neutral with regard to jurisdictional claims in published maps and institutional affiliations.

Copyright: (C) 2022 by the authors. Licensee MDPI, Basel, Switzerland. This article is an open access article distributed under the terms and conditions of the Creative Commons Attribution (CC BY) license (https:// creativecommons.org/licenses/by/ $4.0 /)$.

\begin{abstract}
This research presents a new fractional-order discrete-time susceptible-infected-recovered (SIR) epidemic model with vaccination. The dynamical behavior of the suggested model is examined analytically and numerically. Through using phase attractors, bifurcation diagrams, maximum Lyapunov exponent and the $0-1$ test, it is verified that the newly introduced fractional discrete SIR epidemic model vaccination with both commensurate and incommensurate fractional orders has chaotic behavior. The discrete fractional model gives more complex dynamics for incommensurate fractional orders compared to commensurate fractional orders. The reasonable range of commensurate fractional orders is between $\gamma=0.8712$ and $\gamma=1$, while the reasonable range of incommensurate fractional orders is between $\gamma_{2}=0.77$ and $\gamma_{2}=1$. Furthermore, the complexity analysis is performed using approximate entropy $(A p E n)$ and $C_{0}$ complexity to confirm the existence of chaos. Finally, simulations were carried out on MATLAB to verify the efficacy of the given findings.
\end{abstract}

Keywords: discrete SIR epidemic model; commensurate order; incommensurate order; chaos; complexity

\section{Introduction}

Epidemiology is a topic of research in the biological sciences, which explores all the elements that influence whether there are diseases and disorders. Over the last few years, the outbreaks of several diseases such as SARS, Ebola, and COVID-19 have increased the attention of many researchers to the study of epidemiology. In this regard, the construction of infectious diseases mathematical models and discussion of their dynamics are very significant, since these models are used to aid prevent and control diseases. Numerous studies on mathematical epidemic models have been examined for a variety of diseases. These models may be classified as either continuous-time [1-5] or discrete-time models. Differential equations are used to create continuous-time models, while difference equations are used to formulate discrete-time models. Recently, discrete models have been extensively employed to evaluate and study infectious diseases as opposed to continuous models since epidemic data are available during discrete time periods. Furthermore, the discrete-time epidemic models display more complicated dynamics than the continuous-time epidemic models. Numerous significant types of discrete models may be found in [6-9].

Vaccination is a significant component of disease prevention strategies across the globe. A vaccine's effectiveness is based on its ability to reduce the number of susceptible individuals in order to prevent infectious diseases from spreading through the population [10]. The effect of vaccination on the dynamics of an epidemic model has been studied 
by adding a component for the vaccinated individuals to the epidemic model and obtaining the epidemic model with vaccination. For example, in [11] the authors studied the dynamical behavior of a discrete SIR epidemic model with a constant vaccination strategy. The stability and bifurcations of a discrete susceptible-infected-susceptible (SIS) epidemic model with vaccination were investigated in [12], while Xiang et al. in [13] developed a discrete SIRS model that included vaccination and examined its dynamical behavior.

Fractional calculus is a subject in mathematical analysis, where it can be considered as a generalization of integer calculus [14-28]. Despite this, only in the past decades has it been extensively examined, owing to its broad range of use in many areas. Fractional-order derivatives are relatively more accurate than integer-order derivatives because they serve as an effective tool for describing the memory effect throughout all types of processing and materials [29]. Memory effects refer to the fact that the states of systems with a fractional order are determined by all previous states. Over the last several years, numerous academics have concentrated their efforts on discrete fractional calculus [30-32]. Following the proposal of that special field, researchers have become more interested in its applications in neural networks, physics, biology, etc. Meanwhile, since Wu et al. [33] suggested the first chaotic fractional logistic map, the dynamics and control of various fractional-order chaotic discrete-time systems have been intensively studied [34-38].

Fractional-order derivatives have been extensively used in epidemiology, with the majority of these studies focusing on SIR-type models [39-44]. In [45], Selvam et al. investigated the stability of the fractional-order SIR epidemic model using a discretization process, whereas Naik in [46] studied the global dynamics of a fractional-order SIR epidemic model with memory. The analysis and numerical solution of a novel fractional-order SIR dengue model was investigated in [47]. In [48], the stability analysis and bifurcation control for a delayed fractional-order SIR epidemic model with incommensurate order was considered. It is worth noting that all of these studies are based on continuous-time systems. The motivation behind this work, particularly with the current spread of the COVID-19 pandemic, is to assess the benefits of the fractional discrete epidemic model in the field of epidemiology. So far, to the best of our knowledge, the dynamic analysis of a fractional-order discrete-time epidemic model with vaccination based on a Caputo-like difference operator has not yet been investigated. This piqued our interest and inspired us to study the phenomenon and investigate the behavior of a fractional SIR epidemic model with vaccination when the fractional orders are commensurate and incommensurate.

The goal of this article is to contribute to the field of epidemiology by introducing a novel discrete-time SIR epidemic model with vaccination with both commensurate and incommensurate fractional orders. The work is organized as follows. In Section 2, we present the integer-order form of the discrete SIR model and we recast the mathematical model to take the form of the fractional-order discrete SIR model. In Section 3, we look at how the range and type of chaotic behaviors are affected by commensurate and incommensurate fractional orders. Furthermore, we use the $0-1$ test method to distinguish between regular and chaotic behavior. In Section 4 , we use the $C_{0}$ complexity and approximate entropy $(A p E n)$ to analyze the complexity of the proposed discrete SIR epidemic model. Throughout the paper, numerical simulations are used to demonstrate and verify the results.

\section{Mathematical Model}

In this section, we introduce the discrete SIR model as an integer-order discrete system, and then we reformulate it as a fractional-order discrete system by employing the Caputoleft difference operator. 


\subsection{Integer-Order Discrete Model}

Consider the following description of the SIR epidemic model described in [49], in which the population densities $S_{m}, I_{m}$, and $R_{m}$ represent the number of susceptible, infected, and recovered individuals at time $m$, respectively:

$$
\left\{\begin{array}{l}
S_{m+1}=S_{M}-\frac{\alpha}{N} I_{m} S_{m}+\beta\left(R_{m}+I_{m}\right), \\
I_{m+1}=\frac{\alpha}{N} I_{m} S_{m}+(1-\beta-\sigma) I_{m}, \\
R_{m+1}=(1-\beta) R_{m}+\sigma I_{m},
\end{array}\right.
$$

where $\alpha>0$ is the contact number, $0<\beta<1$ is is the probability of birth, and $0<\sigma<1$ is the probability of recovery. The flow diagram for model (3) is seen in Figure 1.

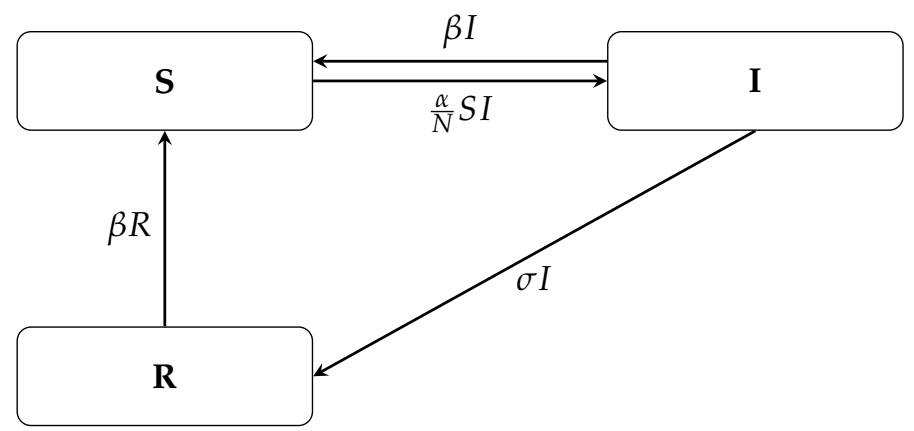

Figure 1. Flow chart of the SIR epidemic model (1).

In [50], the authors focused on the dynamics of an SIR epidemic model by incorporating vaccination in the model (1). The proportion of persons who had been vaccinated was represented by the parameter $p$, where $0<p<1$; and the remainder had a risk of susceptibility to infection in the proportion $1-p$. The following is the general SIR epidemic model with vaccination, which takes the following form:

$$
\left\{\begin{array}{l}
S_{m+1}=(1-p) S_{m}-\frac{\alpha}{N} I_{m} S_{m}+\beta\left(R_{m}+I_{m}\right), \\
I_{m+1}=\frac{\alpha}{N} I_{m} S_{m}+(1-\beta-\sigma) I_{m}, \\
R_{m+1}=(1-\beta) R_{m}+\sigma I_{m}+p S_{m},
\end{array}\right.
$$

$\left(S_{0}, I_{0}, R_{0}\right)$ are initial conditions that are positive real values, with $S_{0}+I_{0}+R_{0}=N$. N is the total population size. Furthermore, by employing the relation $S_{m}+I_{m}+R_{m}=N$, we replace $R_{m}$ by $N-S_{m}-I_{m}$ and we get the following system:

$$
\left\{\begin{array}{l}
S_{m+1}=(1-p) S_{m}-\frac{\alpha}{N} I_{m} S_{m}+\beta\left(N-S_{m}\right), \\
I_{m+1}=\frac{\alpha}{N} I_{m} S_{m}+(1-\beta-\sigma) I_{m}, \\
R_{m+1}=(1-\beta) R_{m}+\sigma I_{m}+p S_{m},
\end{array}\right.
$$

Observing that the two equations concerning $(S, I)$ in the SIR epidemic model (3) do not contain $R$, they are thus independent of the third one. As a result, the three-dimensional 
SIR epidemic model (3) can be reduced to a two-dimensional model, which results in the following equivalent system:

$$
\left\{\begin{array}{l}
S_{m+1}=(1-p) S_{m}-\frac{\alpha}{N} I_{m} S_{m}+\beta\left(N-S_{m}\right), \\
I_{m+1}=\frac{\alpha}{N} I_{m} S_{m}+(1-\beta-\sigma) I_{m},
\end{array}\right.
$$

when $\alpha=4, \beta=0.8, \sigma=0.1, p=0.005$, and $N=100$, the discrete SIR epidemic model with vaccination (4) exhibits a chaotic attractor as shown in Figure 2.

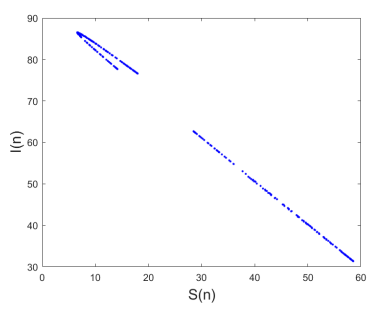

(a)

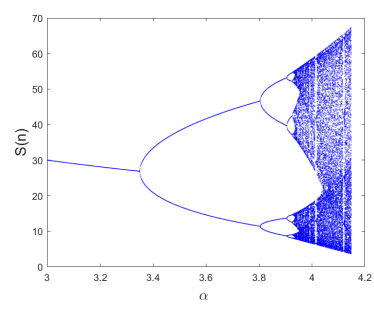

(b)

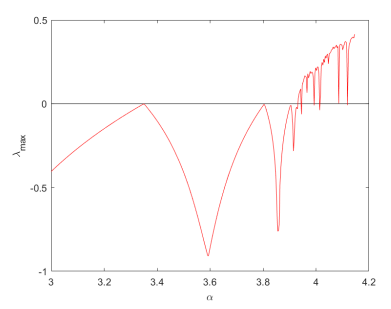

(c)

Figure 2. (a) Phase space of the discrete SIR epidemic model with vaccination (4) for $\alpha=4, \beta=0.8$, $\sigma=0.1, p=0.005, N=100$, and initial conditions $(S(0), I(0))=(70,30)$. (b) Bifurcation diagram of the SIR epidemic model (4) versus $\alpha$. (c) The maximum Lyapunov exponents.

\subsection{Fractional-Order Discrete Model}

In this study, a discrete-time SIR epidemic model with vaccination with both commensurate and incommensurate orders is considered. Herein, the first-order difference of the SIR model (4) is formulated as:

$$
\left\{\begin{array}{l}
\Delta S(m)=-p S(m)-\frac{\alpha}{N} I(m) S(m)+\beta(N-S(m)), \\
\Delta I(s)=\frac{\alpha}{N} I(m) S(m)-(\beta+\sigma) I(m)
\end{array}\right.
$$

As mentioned above, we use the Caputo-left difference operator to create the fractional version of the discrete-time SIR epidemic model with vaccination. The Caputo-left difference operator is defined as follows:

Definition 1. [51] The $\gamma$-Caputo fractional difference operator for a function $h(s)$, is defined by

$$
{ }^{c} \Delta_{a}^{\gamma} h(s)=\Delta_{a}^{-(m-\gamma)} \Delta^{m} h(s)=\frac{1}{\Gamma(m-\gamma)} \sum_{\tau=a}^{s-(m-\gamma)}(s-\tau-1)^{(m-\gamma-1)} \Delta^{m} h(\tau),
$$

where $s \in \mathbb{N}_{a+m-\gamma}, m=\lceil\gamma\rceil+1$, and $\gamma \notin \mathbb{N} .(s-1-\tau)^{(m-\gamma-1)}$ and $\Delta^{m} h(\tau)$ are the falling factorial function and the $m$-th integer difference operator, respectively, which are defined as

$$
(s-\tau-1)^{(m-\gamma-1)}=\frac{\Gamma(s-\tau)}{\Gamma(s-\tau-m+\gamma+1)},
$$

and

$$
\Delta^{m} h(s)=\Delta\left(\Delta^{m-1} h(s)\right)=\sum_{k=0}^{m}\left(\begin{array}{c}
m \\
k
\end{array}\right)(-1)^{m-k} h(s+k), \quad s \in \mathbb{N}_{a}
$$

Remark 1. If we consider that $m=1$, we can define the $\gamma$-Caputo fractional difference operator by

$$
{ }^{c} \Delta_{a}^{\gamma} h(s)=\Delta_{a}^{-(1-\gamma)} \Delta h(s)=\frac{1}{\Gamma(1-\gamma)} \sum_{\tau=a}^{s-(1-\gamma)}(s-\tau-1)^{(-\gamma)} \Delta h(\tau), \quad s \in \mathbb{N}_{a+1-\gamma}
$$


Definition 2. [30] The $\gamma$-th fractional sum for a function $h$ is defined as

$$
\Delta_{a}^{-\gamma} h(s)=\frac{1}{\Gamma(\gamma)} \sum_{\tau=a}^{s-\gamma}(s-1-\tau)^{(\gamma-1)} h(\tau),
$$

with $s \in \mathbb{N}_{a+\gamma}, \gamma>0 . N_{a}=\{a, a+1, a+2, \ldots\}$ is a time scale, $a \in \mathbb{R}$.

Now, the discrete SIR epidemic model with vaccination (4) may be expressed in fractional order as follows:

$$
\left\{\begin{array}{l}
{ }^{C} \Delta_{a}^{\gamma_{1}} S(s)=-p S\left(s-1+\gamma_{1}\right)-\frac{\alpha}{N} I\left(s-1+\gamma_{1}\right) S\left(s-1+\gamma_{1}\right)+\beta\left(N-S\left(s-1+\gamma_{2}\right)\right), \\
{ }^{C} \Delta_{a}^{\gamma_{2}} I(s)=\frac{\alpha}{N} I\left(s-1+\gamma_{2}\right) S\left(s-1+\gamma_{2}\right)-(\beta+\sigma) I\left(s-1+\gamma_{2}\right),
\end{array}\right.
$$

where $\gamma_{i(i=1,2,3)}$ are the fractional order such that $0<\gamma_{i}<1$. Note that if $\gamma_{1}=\gamma_{2}$, then system (11) is referred to as a commensurate fractional-order system, whereas it is referred to as an incommensurate fractional-order system if $\gamma_{1} \neq \gamma_{2}$.

\section{Dynamical Analysis and Numerical Simulations}

In this section, we investigate whether the previously suggested fractional-order discrete-time SIR epidemic model with vaccination (11) exhibits chaotic behavior in both the commensurate and incommensurate fractional orders. This study is conducted utilizing many numerical techniques, including bifurcation diagrams, Lyapunov exponent computations, plotting of phase portraits in $S-I$ projection and applying the $0-1$ method.

In order to show these, we first present a theorem that enables us to obtain the numerical formula for the discrete fractional model that is subsequently discussed.

Theorem 1. [52] For the fractional difference equation

$$
\left\{\begin{array}{l}
{ }^{C} \Delta_{a}^{\gamma_{i}} y(s)=g\left(s+\gamma_{i}-1, y\left(s+\gamma_{i}-1\right)\right) \\
\Delta^{k} y(s)=y_{k}, \quad m=\left\lceil\gamma_{i}\right\rceil+1, \quad k=0,1, \ldots, m-1,
\end{array}\right.
$$

the unique solution of this initial value problem (1) is given by

$$
y(s)=y_{0}(s)+\frac{1}{\Gamma\left(\gamma_{i}\right)} \sum_{\tau=a+m-\gamma_{i}}^{s-\gamma_{i}}(s-\sigma(\tau))^{\left(\gamma_{i}-1\right)} g\left(\tau+\gamma_{i}-1, y\left(\tau+\gamma_{i}-1\right)\right), \quad s \in N_{a+m},
$$

where

$$
y_{0}(s)=\sum_{k=0}^{m-1} \frac{(s-a)^{k}}{\Gamma(k+1)} \Delta_{k} y(a)
$$

According to Theorem 1, the numerical formula of the fractional discrete-time SIR epidemic model (11) is designed as:

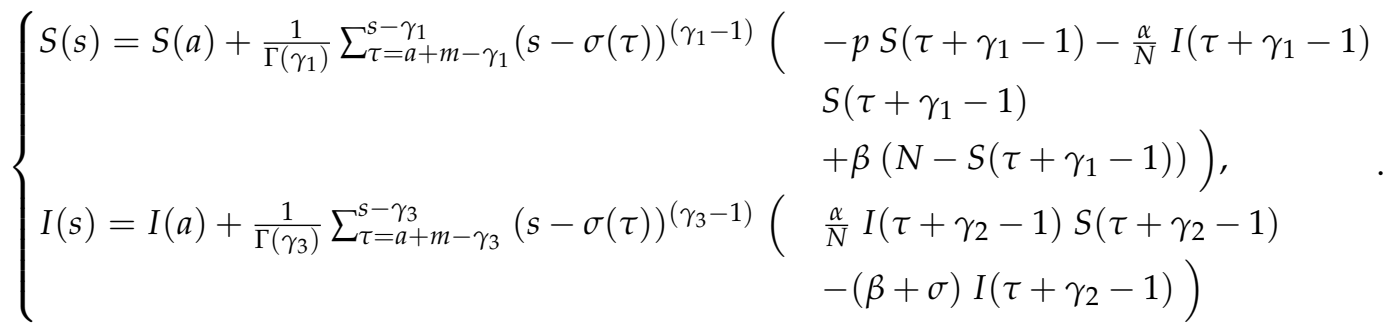


Take $a=0$ and since $(s-\tau+1)^{(\gamma-1)}$ is equal to $\Gamma(s-\tau) / \Gamma(s-\tau-\gamma+1)$, it follows from Theorem 1 that the numerical formula for (11) is obtained as:

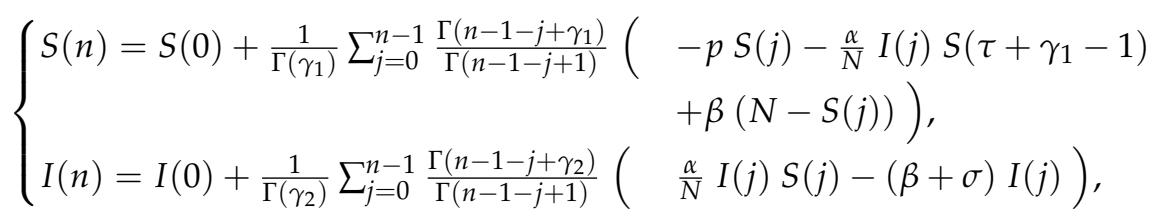

where $S(0)$ and $I(0)$ are the initial conditions. This is a novel type of fractional-order discrete-time SIR epidemic model with vaccination that possesses "memory effects". As seen in Equation (16), the states $S(n)$ and $I(n)$ are dependent on all previous variables $S(0), S(1), \ldots, S(n-1)$, and $I(0), I(1), \ldots, I(n-1)$.

\subsection{Commensurate Fractional Order}

\subsubsection{Bifurcation Diagram and Maximum LEs}

To investigate the dynamic of the commensurate fractional-order discrete-time SIR epidemic model with vaccination given in (11) regarding the fractional order $\gamma=\gamma_{1}=\gamma_{2}$, we set the parameters $N=100, \beta=0.8, \sigma=0.1, p=0.005$, and initial conditions $(S(0), I(0))=(70,30)$. Figure 3 displays the phase space of model (11) for $\gamma=1$. Figure 4 shows the states of the system for 150 points. Take note that when $\gamma=1$, the fractional discrete model (11) refers to the integer-order model. Now, using the same initial condition and the same system parameters, the fractional discrete model for different fractional orders $\gamma$ is presented in Figure 5. We see that as $\gamma$ decreases, the phase portrait changes its shape between chaotic and periodic trajectories, whereas if we continue to decrease $\gamma$, the states of the fractional discrete model diverge to infinity.

The bifurcation diagrams of the fractional discrete model (11) where $\alpha$ varies in the interval $[3,4.5]$ is presented in Figure 6. Obviously, decreasing the fractional order has an effect on the interval in which chaos occurs, and the bifurcation diagram progressively shifts to the right. For example, when $\gamma=0.98$, chaos begins to appear at $\alpha=3.915$ and the maximum chaotic range is reached at $\alpha=4.128$, whereas when $\gamma=0.91$, chaos begins to appear at $\alpha=3.849$ and the maximum chaotic range is reached at $\alpha=4.047$.

To further study the influence of the commensurate fractional order on the dynamics of the fractional discrete-time SIR epidemic model (11), we plotted the bifurcation diagram for $\gamma$ as a critical parameter and the findings are presented in Figure 6a. We notice that the fractional discrete SIR epidemic model (11) contains chaos and that the fractional order $\gamma$ has an influence on the system's dynamics. When $\gamma<0.8712$, the system (11) exhibits a transient state, which means that until a minimum number of iterations is reached, the proposed system's states approach a limited attractor before diverging to infinity.

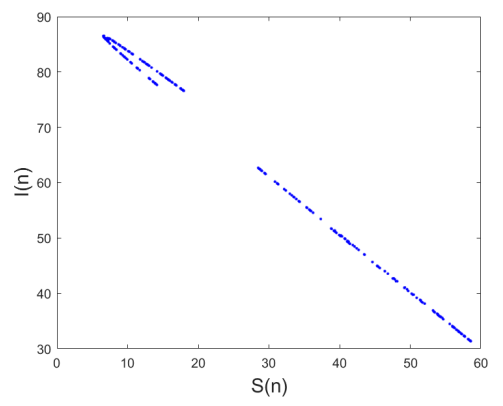

Figure 3. Phase portrait of the fractional SIR epidemic model in (11) with $\gamma=1$ for $N=100, \beta=0.8$, $\sigma=0.1, p=0.0005$, and initial conditions $(S(0), I(0))=(70,30)$. 

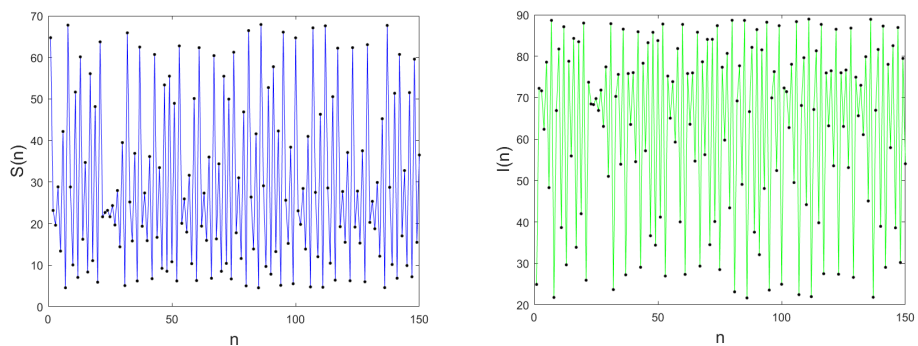

(a)
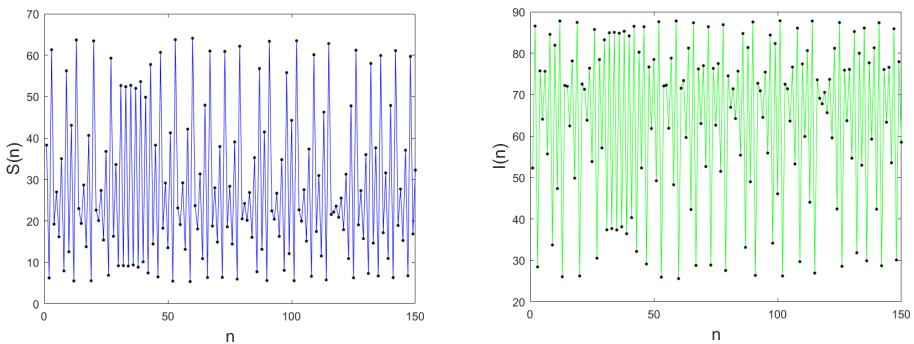

(b)
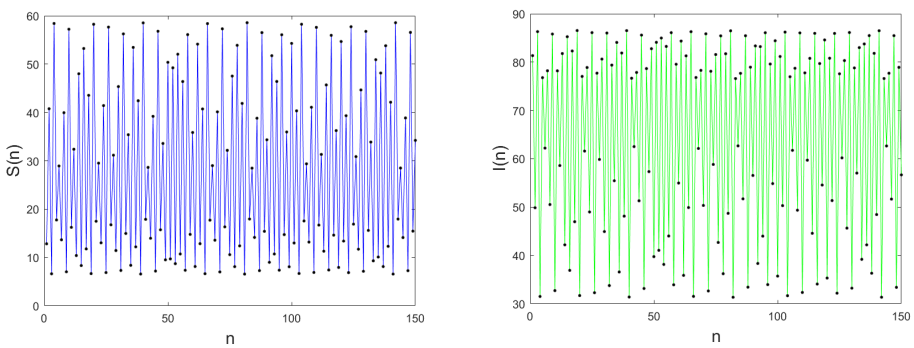

(c)

Figure 4. Time evolution of states for (a) $\gamma=0.89$, (b) $\gamma=0.93$, and (c) $\gamma=1$.
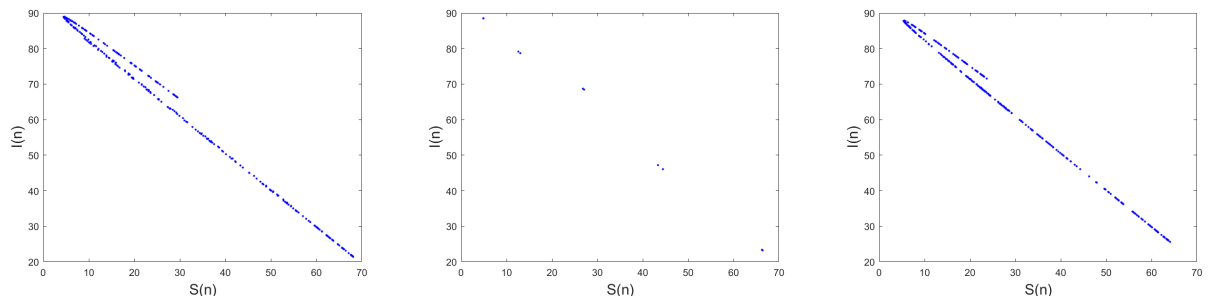

(a) $\gamma=0.89$

(b) $\gamma=0.91$

(c) $\gamma=0.93$
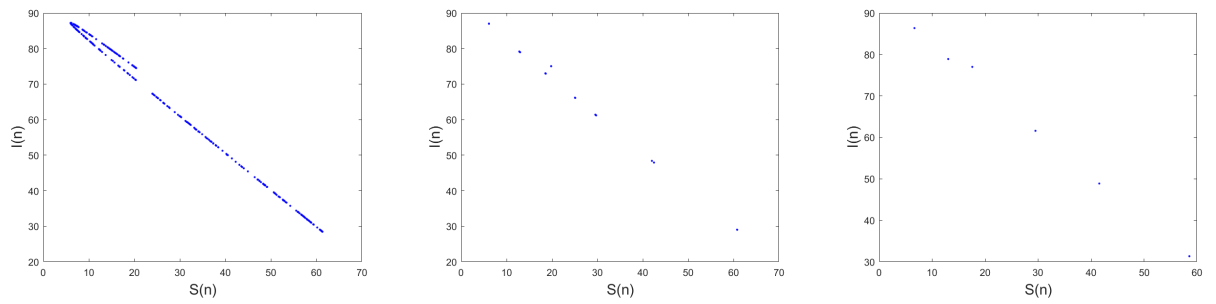

(d) $\gamma=0.96$

(e) $\gamma=0.966$

(f) $\gamma=0.99$

Figure 5. Phase portrait of the fractional SIR epidemic model (11) with commensurate fractional-order values for $N=100, \beta=0.8, \sigma=0.1, p=0.005$, and initial conditions $(S(0), I(0))=(70,30)$. 


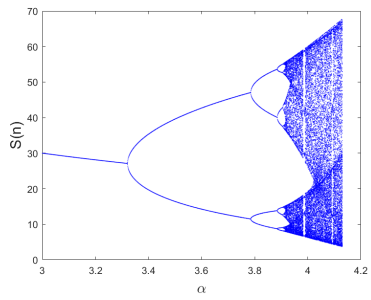

(a) $\gamma=0.98$

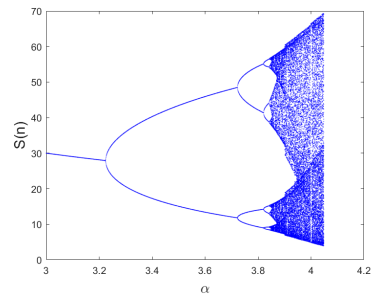

(b) $\gamma=0.91$

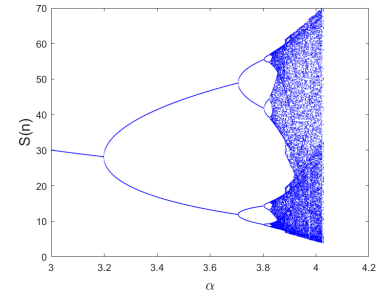

(c) $\gamma=0.89$

Figure 6. Bifurcation diagrams of the fractional discrete SIR model (11) for different commensurate fractional-order values.

An important tool used in fractional discrete systems is the Lyapunov exponents, which are used in conjunction with bifurcation diagrams to show chaos. As shown in [52], the Jacobian matrix algorithm is used to compute or estimate the maximum Lyapunov exponent, which is calculated in a similar way to the states in the fractional-order discrete model (11). The tangent map $J_{i}$ is defined as follows:

$$
J_{i}=\left(\begin{array}{ll}
e_{1} & e_{2} \\
f_{1} & f_{2}
\end{array}\right)
$$

where

$$
\begin{aligned}
& e_{1}(n)=e_{1}(0)+\frac{1}{\Gamma\left(\gamma_{1}\right)} \sum_{j=0}^{n-1} \frac{\Gamma\left(n-1-j+\gamma_{1}\right)}{\Gamma(n-1-j+1)}\left(-p e_{1}(j)-\frac{\alpha}{N}\left(e_{1}(j) I(j)+f_{1}(j) S(j)\right)\right. \\
& \left.-\beta e_{1}(j)\right), \\
& e_{2}(n)=e_{2}(0)+\frac{1}{\Gamma\left(\gamma_{1}\right)} \sum_{j=0}^{n-1} \frac{\Gamma\left(n-1-j+\gamma_{1}\right)}{\Gamma(n-1-j+1)}\left(-p e_{2}(j)-\frac{\alpha}{N}\left(e_{2}(j) I(j)+f_{2}(j) S(j)\right)\right. \\
& \left.-\beta e_{2}(j)\right), \\
& f_{1}(n)=f_{1}(0)+\frac{1}{\Gamma\left(\gamma_{2}\right)} \sum_{j=0}^{n-1} \frac{\Gamma\left(n-1-j+\gamma_{2}\right)}{\Gamma(n-1-j+1)}\left(\frac{\alpha}{N}\left(e_{1}(j) I(j)+f_{1}(j) S(j)\right)-(\beta+\sigma) f_{1}(j)\right), \\
& f_{2}(n)=f_{2}(0)+\frac{1}{\Gamma\left(\gamma_{2}\right)} \sum_{j=0}^{n-1} \frac{\Gamma\left(n-1-j+\gamma_{2}\right)}{\Gamma(n-1-j+1)}\left(\frac{\alpha}{N}\left(e_{2}(j) I(j)+f_{2}(j) S(j)\right)-(\beta+\sigma) f_{2}(j)\right) .
\end{aligned}
$$

Then, the Lyapunov exponents can be given by :

$$
\lambda_{k}\left(x_{0}\right)=\lim _{i \rightarrow \infty} \frac{1}{i} \ln \left|\lambda_{k}^{(i)}\right| \text { for } k=1,2 .
$$

where $\lambda_{k}$ are the eigenvalues of the Jacobian matrix $J_{i}$.

The method described above can be turned into a MATLAB script to calculate the largest LE of the fractional-order discrete-time SIR epidemic model with vaccination (11). Because it is difficult to predict the behavior of the system using analytical techniques, we must rely on approximate numerical approaches, which can be achieved by using MATLAB software. For further information on how to use numerical simulation of some chaotic systems using MATLAB, see the link in [53]. The results of the MLE for system with the same parameters and with initial conditions $(S(0), I(0))=(70,30)$ are shown in Figure $7 \mathrm{~b}$. As can be seen, the system has positive LE, which shows that the MLE is compatible with the bifurcation diagram depicted in Figure 7a. We can also see that some values of Lyapunov exponents are negative, meaning that the system has periodic attractors, and this is illustrated further by the phase portraits plotted in Figure 5. 


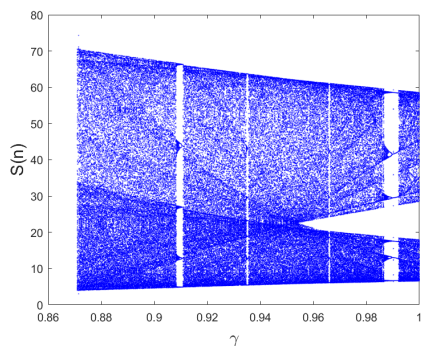

(a)

Figure 7. (a) Bifurcation diagram versus $\gamma$ of the fractional discrete SIR model (11) with $\alpha=4$. (b) The maximum Lyapunov exponent.

\subsubsection{The $0-1$ Test}

To test chaotic behavior in nonlinear systems, one can use a $0-1$ test method [54]. Unlike the Lyapunov exponent approach, the $0-1$ test acts directly on the time sequence, therefore eliminating the necessity for phase reconstruction. In particular, this technique operates on finite points $(S(n))_{n=1, \ldots, N}$ and an arbitrary number $c \in(0, \pi)$. According to the time series $(S(n))$, two terms referred to as the translation variables can be defined for $n=\overline{1, N}$ as $p_{c}(n)=\sum_{k=1}^{n} S(k) \cos (k c)$ and $q_{c}(n)=\sum_{k=1}^{n} S(k) \sin (k c)$. We represent the mean square displacement by

$$
M_{\mathcal{C}}(n)=\lim _{N \rightarrow \infty} \frac{1}{N} \sum_{k=1}^{N}\left[\left(p_{c}(n+k)-p_{c}(k)\right)^{2}+\left(q_{c}(n+k)-q_{c}(k)\right)^{2}\right], \quad n \leq \frac{N}{10} .
$$

The asymptotic growth rate $K_{c}$ is given by the definition

$$
K_{c}=\lim _{n \rightarrow \infty} \frac{\log M_{c}(n)}{\log n} \text {. }
$$

The asymptotic growth rate $K=\operatorname{median}\left(K_{c}\right)$ or the plotting of $p_{c}$ and $q_{c}$ on the $p-q$ plane may be used to assess if chaos occurs on the fractional discrete SIR epidemic model. This means that the dynamics of the proposed fractional discrete SIR epidemic model are nonchaotic when $K$ is close to 0 and the behavior of trajectory in the $\left(p_{c}-q_{c}\right)$ plane is bounded; on the other hand, when $K$ is close to 1 and the trajectory in the $\left(p_{c}-q_{c}\right)$ plane exhibits Brownian-like behavior, the dynamics of the fractional discrete SIR epidemic model are chaotic.

The $0-1$ test of the fractional discrete-time SIR epidemic model with vaccination (11) is applied directly to the state $S(n)$. Figure 8 shows the $\left(p_{c}-q_{c}\right)$ plots of the fractional discrete SIR epidemic model (11) with commensurate fractional order. Observing the $p_{c}$ and $q_{c}$ trajectories, it is clear that the $p_{c}$ and $q_{c}$ trajectories display Brownian-like behavior when $\gamma=1$ and $\gamma=0.94$, indicating that the fractional SIR model is chaotic. Otherwise, when $\gamma_{3}=0.99$ and $\gamma_{3}=0.966$, the trajectories in the $\left(p_{c}-q_{c}\right)$ plane exhibit a bounded behavior, so the SIR model with commensurate order yields regular dynamics. 


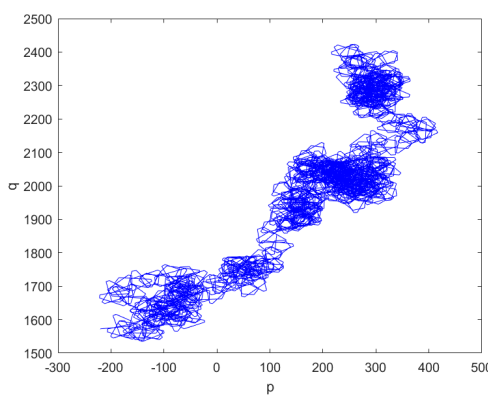

(a) $\gamma=0.94$

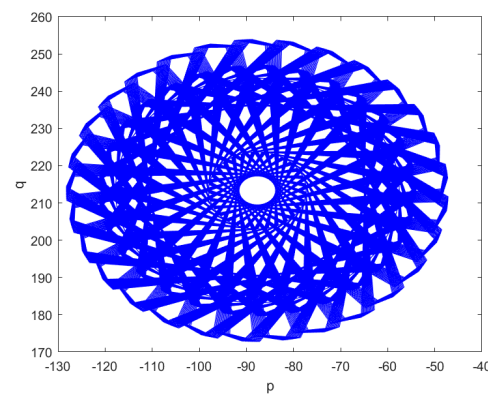

(c) $\gamma=0.99$

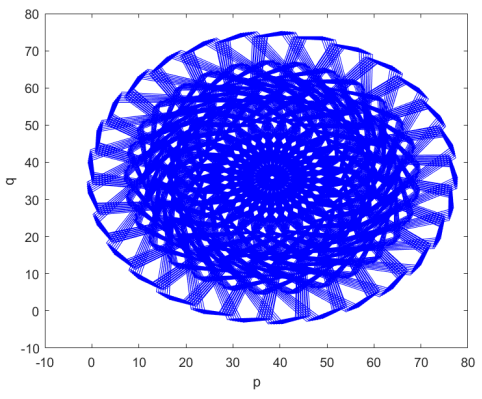

(b) $\gamma=0.966$

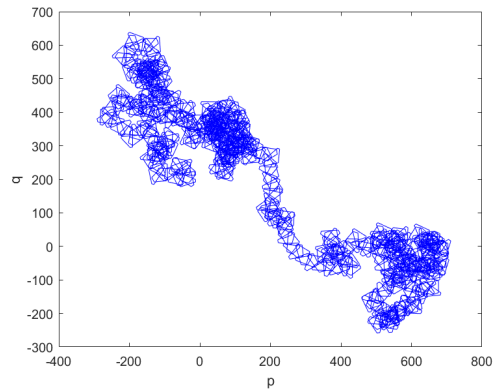

(d) $\gamma=1$

Figure 8. The $p-q$ trajectories of the $0-1$ test of the fractional discrete SIR epidemic model (11) with commensurate orders $\left(\gamma_{1}=\gamma_{2}\right)$.

\subsection{Incommensurate Fractional Order}

In the following, the chaotic behavior of the proposed fractional SIR model with incommensurate fractional order (11) are carefully analyzed via the computation of bifurcation diagrams, Lyapunov exponents, phase portraits and 0-1 test method. Similarly, the effects of system parameter $\alpha$ and the fractional-order values on the dynamics of the model are illustrated in detail. We examine the case of incommensurate orders because it is more representative of reality than the case of commensurate orders, as each variable changes and moves independently of the others, implying that the rank of the influencer varies from one equation to another.

\subsubsection{Bifurcation Diagram and Maximum LEs}

Here, we discuss the dynamics of the fractional discrete SIR model (11) by varying $\alpha$ from 3 to 4.5 with the step size $\Delta \alpha=0.001$. Figure 9 displays the bifurcation diagrams for the fractional order values $\left(\gamma_{1}, \gamma_{2}\right)=(0.94,0.97),\left(\gamma_{1}, \gamma_{2}\right)=(0.94,0.99)$, and $\left(\gamma_{1}, \gamma_{2}\right)=$ $(0.99,0.94)$, respectively. From Figure 9 , we see that the system exhibits periodic doubling or flip bifurcation. When $\alpha$ increases from 3, stability begins with a one-period orbit, then periodic orbits of periods 2,4 , and 8 are seen, which eventually evolve into chaos. In addition, we observe that the states of the system (11) are influenced by the fractionalorder values $\gamma_{i}$ and the system parameter $\alpha$, as shown in Figure 9. As can be observed, when the incommensurate fractional orders $\left(\gamma_{1}, \gamma_{2}\right)$ are changed, the interval in which the chaos may be found shifts. Namely, when $\left(\gamma_{1}, \gamma_{2}\right)=(0.94,0.97)$ the system is chaotic at $\alpha \in] 3.822,3.907[\cup] 3.915,4.008[\cup] 4.014,4.055\left[\right.$, while when $\left(\gamma_{1}, \gamma_{2}\right)=(0.99,0.94)$ the system is chaotic at $\alpha \in] 4.023,3.136[\cup] 4.139,4.1754[$. As a consequence of these findings, it can be concluded that the suggested fractional discrete SIR epidemic model (11) with incommensurate fractional orders shows a greater variety of chaotic attractors than the fractional discrete SIR epidemic model with commensurate fractional orders. To further investigate the effect of the incommensurate fractional orders on the dynamics behavior of system (11), Figure 10 displays the bifurcation diagram and the maximum Lyapunov exponents with $\gamma_{1}$ as a bifurcation parameter where the initial conditions are $(S(0), I(0))=$ 
$(70,30)$, the system parameter is $\alpha=4$, and the fractional order is $\gamma_{2}=0.94$. We can see that the states of the system diverge towards infinity as soon as $\gamma_{1}$ goes below 0.8383 and when $\gamma_{1}$ increases, chaos occurs with certain periodic orbits. We can also see that when $\gamma_{1}$ approaches 1, the states become totally periodic. In Figure 10b, the maximum, Lyapunov exponents calculated for the same parameters and initial conditions as in Figure 10a are shown. Obviously, the maximum Lyapunov exponents have a positive number indicating that chaos exists, which agrees with the corresponding bifurcation diagram in Figure 10a.

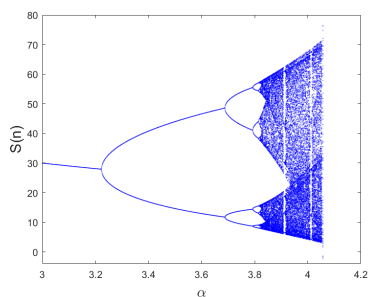

(a) $\gamma_{1}=0.94, \gamma_{2}=0.97$

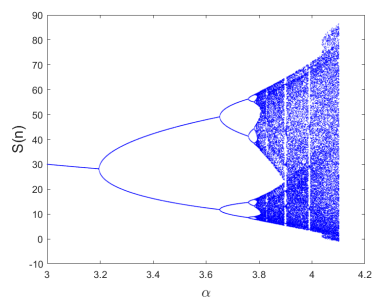

(b) $\gamma_{1}=0.94, \gamma_{2}=0.99$

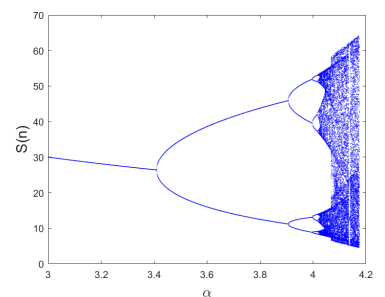

(c) $\gamma_{1}=0.99, \gamma_{2}=0.94$

Figure 9. Bifurcation diagrams of the fractional discrete SIR model (11) for different incommensurate fractional-order values.

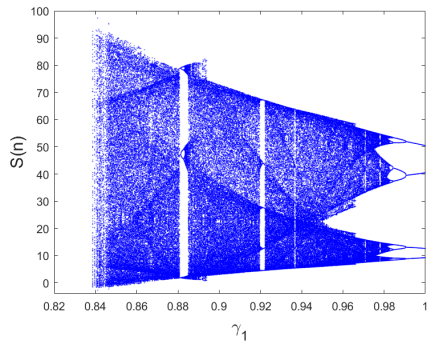

(a)

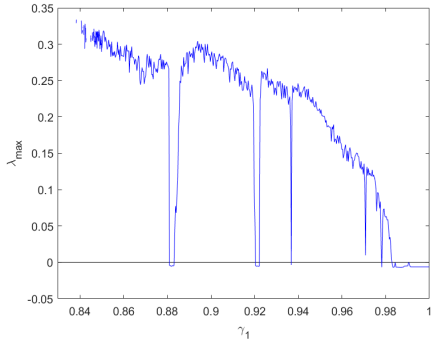

(b)

Figure 10. (a) Bifurcation diagram versus $\gamma_{1}$ of the fractional discrete SIR model (11) for $\alpha=4$ and $\gamma_{2}=0.94$. (b) The maximum Lyapunov exponents.

Now, the dynamic behavior with the variation of the fractional order $\gamma_{2}$ is studied for $\gamma_{1}=0.94$. The bifurcation diagram and its corresponding maximum LE are illustrated in Figure 11. We can see that the dynamical behavior of the fractional discrete SIR epidemic model (11) evolves from periodic to chaos as $\gamma_{2}$ increases. In particular, the proposed SIR model is chaotic when $\left.\left.\gamma_{2} \in\right] 0.888,0.9494[\cup] 0.951,0.979[\cup] 0.9836,1\right]$; where the maximum $\mathrm{LE}$ is positive. The results illustrate that the dynamics behavior of the SIR model is affected by the order $\gamma_{2}$. To illustrate the dynamics of the fractional discrete SIR epidemic model (11) better, phase portraits with different values of $\left(\gamma_{1}, \gamma_{2}\right)$ are presented in Figure 12 From Figure 12, we notice that the proposed SIR model shows different dynamic behaviors for these corresponding different fractional-order values.

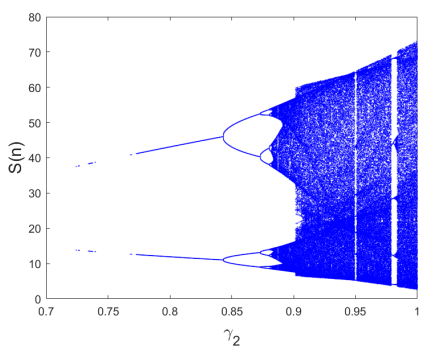

(a)

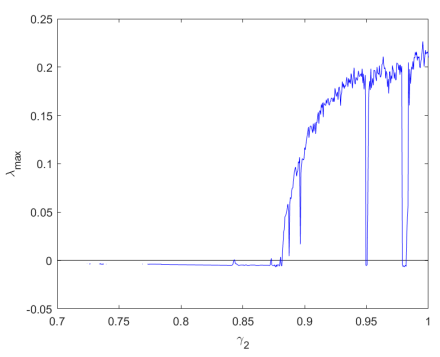

(b)

Figure 11. (a) Bifurcation diagram versus $\gamma_{2}$ of the fractional discrete SIR model (11) for $\alpha=4$ and $\gamma_{1}=0.94$. (b) The maximum Lyapunov exponents. 


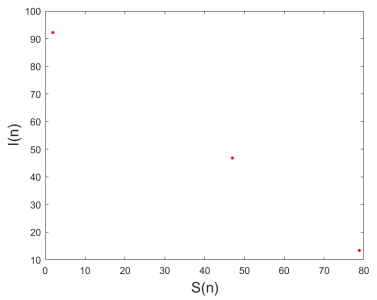

(a) $\left(\gamma_{1}, \gamma_{2}\right)=(0.88,0.94)$

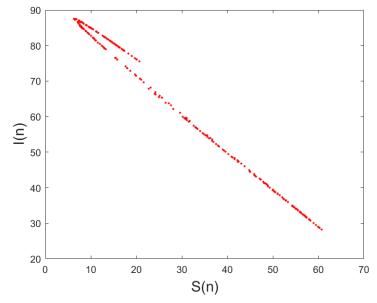

(d) $\left(\gamma_{1}, \gamma_{2}\right)=(0.94,0.91)$

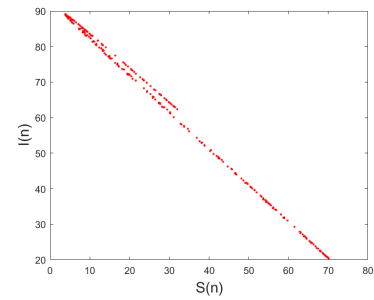

(b) $\left(\gamma_{1}, \gamma_{2}\right)=(0.91,0.94)$

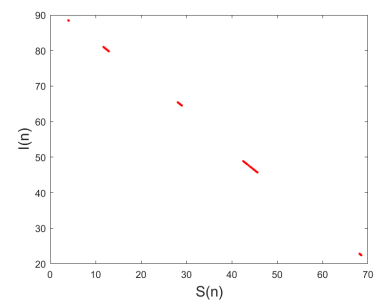

(e) $\left(\gamma_{1}, \gamma_{2}\right)=(0.94,0.983)$

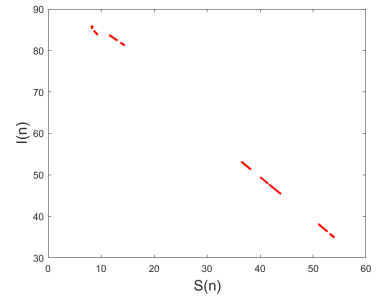

(c) $\left(\gamma_{1}, \gamma_{2}\right)=(0.982,0.94)$

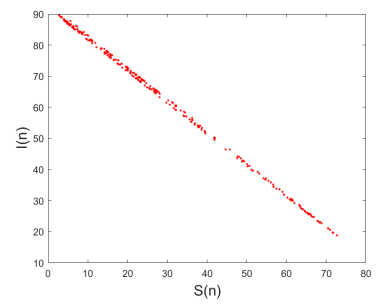

(f) $\left(\gamma_{1}, \gamma_{2}\right)=(0.94,1)$

Figure 12. Phase portrait of the fractional discrete SIR epidemic model with vaccination (11) with incommensurate fractional-order values for $N=100, \beta=0.8, \sigma=0.1, p=0.005$, and initial conditions $(S(0), I(0))=(70,30)$.

To get a better understanding of the influence of the fractional order on the SIR epidemic model, and in light of previous numerical findings, we compare the recent results obtained from the integer-order SIR model with the results obtained from the fractionalorder SIR model, which are presented in Table 1. It can be observed that the maximum number of susceptible cases obtained from the fractional-order SIR model is identical to the maximum number obtained from the integer-order SIR model, which is 70, whereas the minimum number of susceptible cases obtained from the fractional-order SIR model is less than the minimum number obtained from the integer-order SIR model. On the other hand, we see that the maximum and minimum numbers of infected cases predicted in the fractional-order SIR model are not identical to those expected in the integer-order SIR model. This confirms the effect of the fractional order on the SIR epidemic model in predicting the number of susceptible individuals and infected individuals.

Table 1. The minimum and maximum numbers of expected cases for the susceptible class and infected class.

\begin{tabular}{ccc}
\hline Classes & $\begin{array}{c}\text { Integer-Order Model } \\
(\mathbf{m i n}, \mathbf{m a x})\end{array}$ & $\begin{array}{c}\text { Fractional-Order Model } \\
(\mathbf{m i n}, \mathbf{m a x})\end{array}$ \\
\hline Susceptible individuals $(S)$ & $(7,70)$ & $(4,70)$ \\
Infected individuals $(I)$ & $(30,86)$ & $(20,90)$ \\
\hline
\end{tabular}

\subsubsection{The $0-1$ Test}

Similarly to the commensurate fractional orders, the $0-1$ test was used to evaluate the fractional discrete SIR epidemic model (11) with incommensurate fractional order. The translation components $p_{c}$ and $q_{c}$ in the $\left(p_{c}-q_{c}\right)$ plan are illustrated in Figure 13. As can be observed, for $\left(\gamma_{1}, \gamma_{2}\right)=(0.91,0.94)$ and $\left(\gamma_{1}, \gamma_{2}\right)=(0.94,0.91)$, the trajectories $p_{c}$ and $q_{c}$ display Brownian-like behavior, and a bounded behavior for $\left(\gamma_{1}, \gamma_{2}\right)=(0.94,0.983)$ and $\left(\gamma_{1}, \gamma_{2}\right)=(0.982,0.94)$, which confirms that the fractional discrete SIR epidemic model (11) has chaotic attractors for $\left(\gamma_{1}, \gamma_{2}\right)=(0.97,0.94)$ and $\left(\gamma_{1}, \gamma_{2}\right)=(0.97,1)$, and has a regular behavior for $\left(\gamma_{1}, \gamma_{2}\right)=(0.99,0.94)$ and $\left(\gamma_{1}, \gamma_{2}\right)=(0.97,0.9)$. On the other side, Figure 14 depicts the asymptotic growth rate $K$ with fractional orders $\left(\gamma_{1}, \gamma_{2}\right)$, in which $\alpha=4$ and $(S(0), I(0))=(70,30)$. As we can see, for the majority of values of $\gamma_{1}$ and $\gamma_{2}$, the asymptotic growth rate $K$ approaches 1 , implying that the fractional-order discrete SIR 
model with vaccination exhibits a chaotic behavior. These findings support very well the results of the bifurcation diagrams and the maximum Lyapunov exponents obtained before.

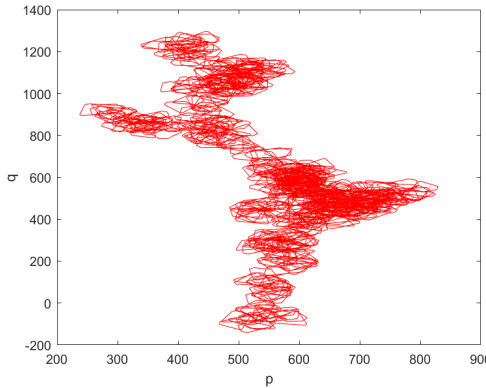

(a) $\left(\gamma_{1}, \gamma_{2}\right)=(0.91,0.94)$

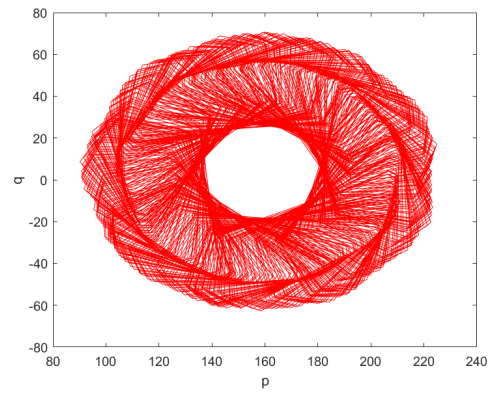

(c) $\left(\gamma_{1}, \gamma_{2}\right)=(0.94,0.983)$

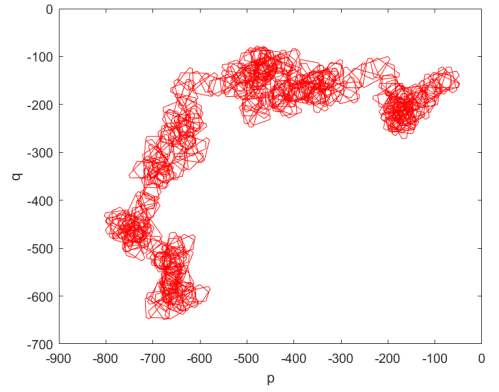

(b) $\left(\gamma_{1}, \gamma_{2}\right)=(0.94,0.91)$

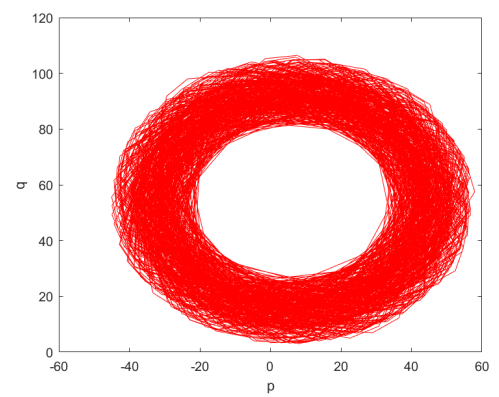

(d) $\left(\gamma_{1}, \gamma_{2}\right)=(0.982,0.94)$

Figure 13. The $p-q$ trajectories of the $0-1$ test of the fractional discrete SIR epidemic model (11) with incommensurate orders $\left(\gamma_{1}, \gamma_{2}\right)$.

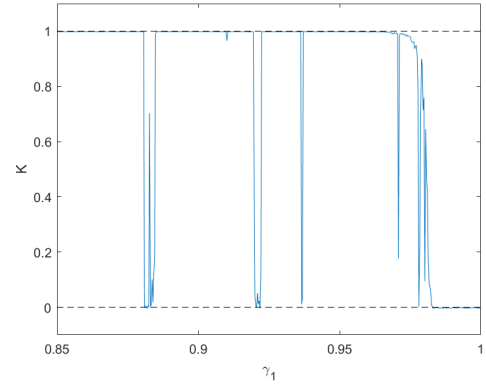

(a)

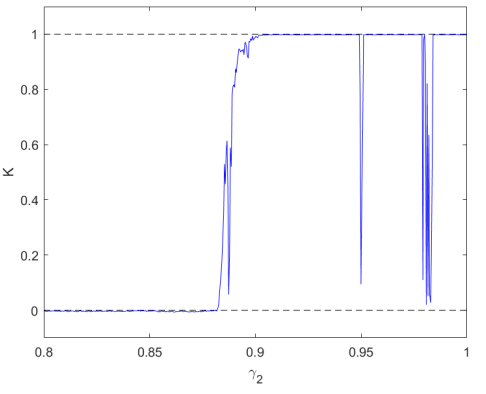

(b)

Figure 14. The asymptotic growth rate $K$ of the fractional discrete SIR epidemic model (11) with $\left(\gamma_{1}, \gamma_{2}\right)$ as critical parameters for (a) $\gamma_{2}=0.94$, (b) $\gamma_{1}=0.94$.

\section{Complexity Analysis of the Fractional Discrete SIR Epidemic Model with Commensurate and Incommensurate Fractional Orders}

For assessing the dynamic characteristics of chaotic systems, one technique is to consider the complexity of the chaotic characteristic. The model becomes more chaotic as the level of complexity increases. In this section, the approximate entropy and the $C_{0}$ complexity algorithm are used to assess the complexity of the fractional-order discrete-time SIR epidemic model with vaccination.

\section{1. $C_{0}$ Complexity}

Based on the inverse Fourier transform, the analysis of the complexity of chaotic systems was carried out using the $C_{0}$ algorithm. It divides the time series of the system into two components, a series of regular and a series of irregular parts, where the series of irregular parts is what we need. For a sequence $[S(0), S(1), \ldots, S(N-1)]$ with a length 
of $N$, and a control parameter $r$, the algorithm process is defined as follows [55]. Firstly, the discrete Fourier transform of $\left\{S_{n}\right\}$ is determined by

$$
X_{N}(k)=\frac{1}{N} \sum_{j=0}^{N-1} S(j) \exp ^{-2 \pi i\left(\frac{k j}{N}\right)}, \quad k=0,1, . ., N-1,
$$

and the mean square value is calculated as $G_{N}=\frac{1}{N} \sum_{k=0}^{N-1}\left|X_{N}(k)\right|^{2}$. We let

$$
\bar{X}_{N}(k)=\left\{\begin{array}{lll}
X_{N}(k) & \text { if } & \left|X_{N}(k)\right|^{2}>r G_{N} \\
0 & \text { if } & \left.X_{N}(k)\right|^{2} \leq r G_{N} .
\end{array}\right.
$$

The inverse Fourier transformation of $\bar{X}_{N}$ is defined as

$$
\bar{x}(j)=\frac{1}{N} \sum_{k=0}^{N-1} \bar{X}_{N}(k) \exp ^{2 \pi i(\stackrel{k j}{N}), \quad j=0,1, . ., N-1 .}
$$

Then, the $C_{0}$ complexity is given by:

$$
C_{0}=\frac{\sum_{j=0}^{N-1}|S(j)-\bar{x}(j)|^{2}}{\sum_{j=0}^{N-1}|S(J)|^{2}} .
$$

The $C_{0}$ complexity of the fractional-order discrete-time SIR epidemic model with vaccination (11) with varying commensurate fractional-order value $\gamma$ and incommensurate fractional-order values $\gamma_{1}, \gamma_{2}$ are calculated and the result is shown in Figure 15. Interestingly, in the case of commensurate fractional-order values $\gamma$, as with the MLE, the $C_{0}$ complexity value of the fractional SIR model increases rapidly when $\gamma_{i}$ decreases. On the other hand, the fractional discrete SIR epidemic model (11) with incommensurate fractionalorder values, in contrast to the case when the model has commensurate fractional-order values, has more complexity when $\gamma_{2}$ approaches 1 . Thus, we can see that the $C_{0}$ algorithm can measure the complexity effectively. Figure 15a illustrates that model (11) has a higher complexity when $\gamma \in(0.9712,0.9082]$. When $\gamma_{1}=0.94$, the high complexity region in Figure $15 \mathrm{c}$ also exists in the range of $\gamma_{2} \in[0.9836,1]$, and the $C_{0}$ complexity increases with the increase of fractional order $\gamma_{2}$.

\subsection{Approximate Entropy (ApEn)}

The approximate entropy is a measure of regularity that quantifies the level of complexity within systems generated by a time series. Generally, time series with larger values of $A p E n$ are considered to be more complex [56]. Note that, the approximate entropy value is dependent on two essential parameters: the similarity tolerance $r$ and the embedding dimension $m$. In this article, we took $m=2$ and $r=0.2 \operatorname{std}(S)$ where $s t d(S)$ represents the standard deviation of the data $S$. Theoretically, the ApEn is calculated as follows:

$$
A p E n=-\Phi^{m+1}(r)+\Phi^{m}(r),
$$

where $\Phi^{m}(r)$ is denoted by

$$
\Phi^{m}(r)=\frac{1}{n-m-1} \sum_{i=1}^{n-m+1} \log C_{i}^{m}(r)
$$

where $C_{i}^{m}(r)=\frac{K}{n-m+1}, i \in[1, n-m+1], K$ is the number of $X(j)$ such that $d(X(j), X(i)) \leq$ $r$, and $X(i)=[x(i), x(i+1), \ldots, x(i+m-1)]$.

The ApEn complexity of the fractional-order discrete-time SIR epidemic model with vaccination (11), with both commensurate and incommensurate orders $\left(\gamma_{1}, \gamma_{2}\right)$ was ana- 
lyzed and the findings are reported in Table 2. It can be seen that the complexity of the fractional discrete SIR epidemic model (11) varies when $\gamma_{i}(i=1,2)$ varies, and the highest $A p E n$ is found when the model is chaotic, which agrees very well with the maximum LE results. As a result, in order to obtain a relatively high structural complexity, we must be cautious when choosing the values of $\gamma_{i}$ in system (11).

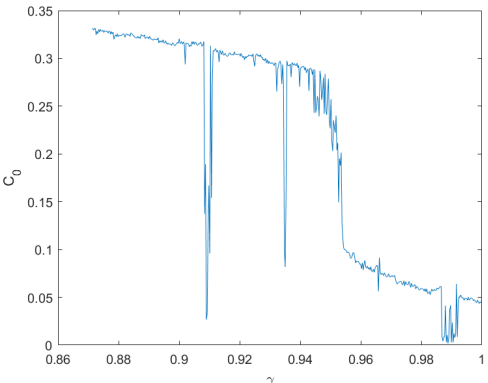

(a)

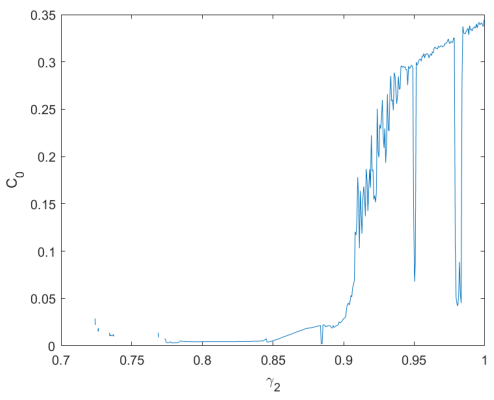

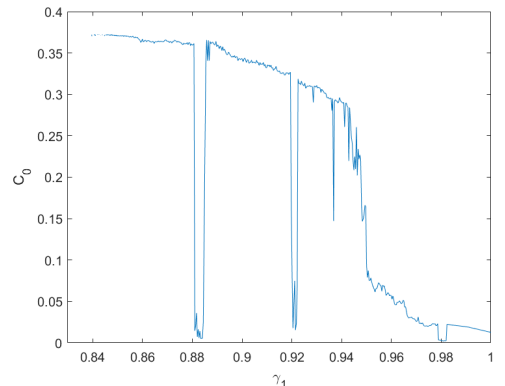

(b)

(c)

Figure 15. $C_{0}$ complexity analysis of the SIR epidemic model (11) for $\alpha=4$ and $(S(0), I(0))=(70,30)$ and with fractional-order values: (a) $\gamma_{1}=\gamma_{2},(\mathbf{b}) \gamma_{1}=0.94$, and (c) $\gamma_{2}=0.94$.

Table 2. Approximate entropy test of the SIR model (11) with different fractional-order values $\gamma_{i}, i=1,2$.

\begin{tabular}{ccc}
\hline$\gamma_{1}$ & $\gamma_{\mathbf{2}}$ & ApEn \\
\hline 1 & 1 & 0.2552 \\
0.99 & 0.99 & 0.0265 \\
0.94 & 0.94 & 0.3718 \\
0.94 & 0.983 & 0.0503 \\
0.982 & 0.94 & 0.1336 \\
0.91 & 0.94 & 0.4056 \\
\hline
\end{tabular}

\section{Conclusions}

In this paper, we dealt with the dynamics of a new fractional-order discrete-time SIR epidemic model with vaccination with commensurate and incommensurate orders. Through phase portraits, bifurcation diagrams, and maximum LEs, the complex dynamics of the proposed system were discussed. In addition, we also calculated the $0-1$ test, approximate entropy $(A p E n)$, and $C_{0}$ complexity of the fractional-order discrete SIR epidemic model for different fractional order values, all of which intended to demonstrate and quantify the complex dynamics of the system. Results indicated that the fractionalorder model is more complex than the integer-order model. Furthermore, we showed that the incommensurate fractional orders have a greater effect on the behavior of the system than when the fractional orders are commensurate. The reasonable range of commensurate fractional orders is between $\gamma=0.8712$ and $\gamma=1$ while the reasonable range of incommensurate fractional orders is between $\gamma_{2}=0.77$ and $\gamma_{2}=1$. Throughout this work, 
numerical simulations were used to explain all the findings. Due to the current spread of the COVID-19 epidemic and the onset of the implementation of various vaccination strategies in various countries, we will attempt to use the fractal properties of most of the COVID-19 series in order to establish a connection between the proposed fractional model and the stochastic self-affine characteristics of the COVID-19 times series in future research. In addition, the determination of the coupled time-fractional differential equations of the proposed fractional model using the Caputo fractional derivative, as well as the study of some complex epidemiological models will be considered in our future research.

Author Contributions: Conceptualization, Z.Y.-H., A.A.; H.J., N.D.A. and Y.W.; methodology, Z.Y.-H., A.A., H.J., N.D.A. and Y.W.; software, Z.Y.-H., A.A., H.J., N.D.A. and Y.W.; validation, Z.Y.-H., A.A., H.J., N.D.A. and Y.W.; formal analysis, Z.Y.-H., A.A., H.J., N.D.A. and Y.W.; investigation, Z.Y.-H., A.A., H.J., N.D.A. and Y.W.; writing-original draft preparation, Z.Y.-H., A.A., H.J., N.D.A. and Y.W.; writing-review and editing, Z.Y.-H., A.A., H.J., N.D.A. and Y.W. All authors have read and agreed to the published version of the manuscript.

Funding: The Deanship of Scientific Research (DSR) at King Abdulaziz University, Jeddah, Saudi Arabia has funded this project, under grant no. (FP-035-43).

Institutional Review Board Statement: Not applicable.

Informed Consent Statement: Not applicable.

Conflicts of Interest: The authors declare no conflict of interest.

\section{References}

1. Lv, W.; Ke, Q.; Li, K. Dynamical analysis and control strategies of an SIVS epidemic model with imperfect vaccination on scale-free networks. Nonlinear Dyn. 2020, 99, 1507-1523. [CrossRef]

2. Din, A.; Khan, A.; Baleanu, D. Stationary distribution and extinction of stochastic coronavirus (COVID-19) epidemic model. Chaos Solitons Fractals 2020, 139, 110036. [CrossRef] [PubMed]

3. Pandey, P.; Chu, Y.M.; Gómez-Aguilar, J.F.; Jahanshahi, H.; Aly, A.A. A novel fractional mathematical model of COVID-19 epidemic considering quarantine and latent time. Results Phys. 2021, 26, 104286. [CrossRef] [PubMed]

4. Jahanshahi, H.; Munoz-Pacheco, J.M.; Bekiros, S.; Alotaibi, N.D. A fractional-order SIRD model with time-dependent memory indexes for encompassing the multi-fractional characteristics of the COVID-19. Chaos Solitons Fractals 2021, 143, 110632. [CrossRef] [PubMed]

5. Zha, T.H.; Castillo, O.; Jahanshahi, H.; Yusuf, A.; Alassafi, M.O.; Alsaadi, F.E.; Chu, Y.M. A fuzzy-based strategy to suppress the novel coronavirus (2019-NCOV) massive outbreak. Appl. Comput. Math. 2021, 20, 160-176.

6. Allen, L.J. Some discrete-time SI, SIR, and SIS epidemic models. Math. Biosci. 1994, 124, 83-105. [CrossRef]

7. Cao, H.; Zhou, Y.; Ma, Z. Bifurcation analysis of a discrete SIS model with bilinear incidence depending on new infection. Math Biosci. Eng. 2013, 10, 1399.

8. Parsamanesh, M.; Mehrshad, S. Stability of the equilibria in a discrete-time sivs epidemic model with standard incidence. Filomat 2019, 33, 2393-2408. [CrossRef]

9. Parsamanesh, M.; Erfanian, M. Stability and bifurcations in a discrete-time SIVS model with saturated incidence rate. Chaos Solitons Fractals 2021, 150, 111178. [CrossRef]

10. Keeling, M.J.; Danon, L. Mathematical modelling of infectious diseases. Br. Med. Bull. 2009, 92, 1. [CrossRef] [PubMed]

11. Rashidinia, J.; Sajjadian, M.; Duarte, J.; Januário, C.; Martins, N. On the dynamical complexity of a seasonally forced discrete SIR epidemic model with a constant vaccination strategy. Complexity 2018, 2018, 7191487. [CrossRef]

12. Parsamanesh, M.; Erfanian, M.; Mehrshad, S. Stability and bifurcations in a discrete-time epidemic model with vaccination and vital dynamics. BMC Bioinform. 2020, 21, 1-5. [CrossRef] [PubMed]

13. Xiang, L.; Zhang, Y.; Huang, J. Stability analysis of a discrete SIRS epidemic model with vaccination. J. Differ. Equations Appl. 2020, 26, 309-327. [CrossRef]

14. Wang, B.; Liu, J.; Alassafi, M.O.; Alsaadi, F.E.; Jahanshahi, H.; Bekiros, S. Intelligent parameter identification and prediction of variable time fractional derivative and application in a symmetric chaotic financial system. Chaos Solitons Fractals 2021, $28,111590$. [CrossRef]

15. Wang, B.; Jahanshahi, H.; Dutta, H.; Zambrano-Serrano, E.; Grebenyuk, V.; Bekiros, S.; Aly, A.A. Incorporating fast and intelligent control technique into ecology: A Chebyshev neural network-based terminal sliding mode approach for fractional chaotic ecological systems. Ecol. Complex. 2021, 47, 100943. [CrossRef]

16. Liu, Z.; Jahanshahi, H.; Gómez-Aguilar, J.F.; Fernandez-Anaya, G.; Torres-Jiménez, J.; Aly, A.A.; Aljuaid, A.M. Fuzzy adaptive control technique for a new fractional-order supply chain system. Phys. Scr. 2021, 96, 124017. [CrossRef] 
17. Wang, H.; Jahanshahi, H.; Wang, M.K.; Bekiros, S.; Liu, J.; Aly, A.A. A Caputo-Fabrizio Fractional-Order Model of HIV / AIDS with a Treatment Compartment: Sensitivity Analysis and Optimal Control Strategies. Entropy 2021, 23, 610. [CrossRef]

18. Wang, Y.L.; Jahanshahi, H.; Bekiros, S.; Bezzina, F.; Chu, Y.M.; Aly, A.A. Deep recurrent neural networks with finite-time terminal sliding mode control for a chaotic fractional-order financial system with market confidence. Chaos Solitons Fractals 2021, 146, 110881. [CrossRef]

19. Li, J.F.; Jahanshahi, H.; Kacar, S.; Chu, Y.M.; Gómez-Aguilar, J.F.; Alotaibi, N.D.; Alharbi, K.H. On the variable-order fractional memristor oscillator: Data security applications and synchronization using a type-2 fuzzy disturbance observer-based robust control. Chaos Solitons Fractals 2021, 145, 110681. [CrossRef]

20. Jahanshahi, H.; Sajjadi, S.S.; Bekiros, S.; Aly, A.A. On the development of variable-order fractional hyperchaotic economic system with a nonlinear model predictive controller. Chaos Solitons Fractals 2021, 144, 110698. [CrossRef]

21. Xiong, P.Y.; Jahanshahi, H.; Alcaraz, R.; Chu, Y.M.; Gómez-Aguilar, J.F.; Alsaadi, F.E. Spectral entropy analysis and synchronization of a multi-stable fractional-order chaotic system using a novel neural network-based chattering-free sliding mode technique. Chaos Solitons Fractals 2021, 144, 110576. [CrossRef]

22. Wang, B.; Jahanshahi, H.; Volos, C.; Bekiros, S.; Khan, M.A.; Agarwal, P.; Aly, A.A. A New RBF Neural Network-Based Fault-Tolerant Active Control for Fractional Time-Delayed Systems. Electronics 2021, 10, 1501. [CrossRef]

23. Wang, B.; Jahanshahi, H.; Bekiros, S.; Chu, Y.M.; Gomez-Aguilar, J.F.; Alsaadi, F.E.; Alassafi, M.O. Tracking control and stabilization of a fractional financial risk system using novel active finite-time fault-tolerant controls. Fractals 2021, 29, $2150155-2150177$. [CrossRef]

24. Chen, S.B.; Soradi-Zeid, S.; Alipour, M.; Chu, Y.M.; Gomez-Aguilar, J.F.; Jahanshahi, H. Optimal control of nonlinear time-delay fractional differential equations with Dickson polynomials. Fractals 2021, 29, 2150079-2150170. [CrossRef]

25. Chen, S.B.; Soradi-Zeid, S.; Jahanshahi, H.; Alcaraz, R.; Gómez-Aguilar, J.F.; Bekiros, S.; Chu, Y.M. Optimal control of time-delay fractional equations via a joint application of radial basis functions and collocation method. Entropy 2020, 22, 1213. [CrossRef] [PubMed]

26. Jahanshahi, H.; Yousefpour, A.; Munoz-Pacheco, J.M.; Kacar, S.; Pham, V.T.; Alsaadi, F.E. A new fractional-order hyperchaotic memristor oscillator: Dynamic analysis, robust adaptive synchronization, and its application to voice encryption. Appl. Math Comput. 2020, 383, 125310. [CrossRef]

27. Soradi-Zeid, S.; Jahanshahi, H.; Yousefpour, A.; Bekiros, S. King algorithm: A novel optimization approach based on variable-order fractional calculus with application in chaotic financial systems. Chaos Solitons Fractals 2020, 132, 109569. [CrossRef]

28. Jahanshahi, H.; Yousefpour, A.; Munoz-Pacheco, J.M.; Moroz, I.; Wei, Z.; Castillo, O. A new multi-stable fractional-order fourdimensional system with self-excited and hidden chaotic attractors: Dynamic analysis and adaptive synchronization using a novel fuzzy adaptive sliding mode control method. Appl. Soft Comput. 2020, 87, 105943. [CrossRef]

29. Podlubny, I. Fractional Differential Equations: An Introduction to Fractional Derivatives, Fractional Differential Equations, to Methods of Their Solution and Some of Their Applications; Elsevier: Amsterdam, The Netherlands, 1998.

30. Atici, F.M.; Eloe, P.W. Discrete fractional calculus with the nabla operator. Electron. J. Qual. Theory Differ. Equ. 2009, 1, 1-99. [CrossRef]

31. Diaz, J.B.; Osler, T.J. Differences of fractional order. Math. Comput. 1974, 28, 185-202. [CrossRef]

32. Anastassiou, G.A. Principles of delta fractional calculus on time scales and inequalities. Math. Comput. Model. 2010, 52, 556-566. [CrossRef]

33. Wu, G.C.; Baleanu, D. Discrete fractional logistic map and its chaos. Nonlinear Dyn. 2014, 75, 283-287. [CrossRef]

34. Khennaoui, A.A.; Ouannas, A.; Bendoukha, S.; Grassi, G.; Wang, X.; Pham, V.T.; Alsaadi, F.E. Chaos, control, and synchronization in some fractional-order difference equations. Adv. Differ. Equ. 2019, 2019, 1-23. [CrossRef]

35. Peng, Y.; He, S.; Sun, K. Chaos in the discrete memristor-based system with fractional-order difference. Results Phys. 2021, 24, 104106. [CrossRef]

36. Khennaoui, A.A.; Ouannas, A.; Bendoukha, S.; Grassi, G.; Lozi, R.P.; Pham, V.T. On fractional-order discrete-time systems: Chaos, stabilization and synchronization. Chaos Solitons Fractals 2019, 119, 150-162. [CrossRef]

37. Ouannas, A.; Khennaoui, A.A.; Odibat, Z.; Pham, V.T.; Grassi, G. On the dynamics, control and synchronization of fractional-order Ikeda map. Chaos Solitons Fractals 2019, 123, 108-115. [CrossRef]

38. Hadjabi, F.; Ouannas, A.; Shawagfeh, N.; Khennaoui, A.A.; Grassi, G. On two-dimensional fractional chaotic maps with symmetries. Symmetry 2020, 12, 756. [CrossRef]

39. El-Saka, H.A. The fractional-order SIR and SIRS epidemic models with variable population size. Math. Sci. Lett. 2013, 2, 195. [CrossRef]

40. Mouaouine, A.; Boukhouima, A.; Hattaf, K.; Yousfi, N. A fractional order SIR epidemic model with nonlinear incidence rate. Adv Differ. Equations 2018, 2018, 1-9. [CrossRef]

41. Momani, S.; Kumar, R.; Srivastava, H.M.; Kumar, S.; Hadid, S. A chaos study of fractional SIR epidemic model of childhood diseases. Results Phys. 2021, 27, 104422. [CrossRef]

42. Owoyemi, A.E.; Sulaiman, I.M.; Mamat, M.; Olowo, S.E. Stability and Bifurcation Analysis in a Fractional-order Epidemic Model with Sub-optimal Immunity, Nonlinear Incidence and Saturated Recovery Rate. IAENG Int. J. Appl. Math. 2021, 51, 3. 
43. Alaoui, A.L.; Tilioua, M.; Sidi Ammi, M.R.; Agarwal, P. Dynamical Analysis of a Caputo Fractional Order SIR Epidemic Model with a General Treatment Function. In Analysis of Infectious Disease Problems (COVID-19) and Their Global Impact; Springer: Singapore, 2021; pp. 17-33.

44. Javeed, S.; Anjum, S.; Alimgeer, K.S.; Atif, M.; Khan, M.S.; Farooq, W.A.; Hanif, A.; Ahmed, H.; Yao, S.W. A Novel Mathematical Model for COVID-19 with Remedial Strategies. Results Phys. 2021, 8, 104248. [CrossRef]

45. Selvam, A.G.; Vianny, D.A. Discrete fractional order SIR epidemic model and it's stability. In Journal of Physics: Conference Series; IOP Publishing: Bristol, UK, 2018; Volume 1139, p. 012008.

46. Naik, P.A. Global dynamics of a fractional-order SIR epidemic model with memory. Int. J. Biomath. 2020, 13, 2050071. [CrossRef]

47. Ahmad, S.; Javeed, S.; Ahmad, H.; Khushi, J.; Elagan, S.K.; Khames, A. Analysis and numerical solution of novel fractional model for dengue. Results Phys. 2021, 28, 104669. [CrossRef]

48. Liu, F.; Huang, S.; Zheng, S.; Wang, H.O. Stability Analysis and Bifurcation Control for a Fractional Order SIR Epidemic Model with Delay. In Proceedings of the 2020 39th Chinese Control Conference (CCC), Shenyang, China, 27-29 July 2020; pp. 724-729.

49. Din, Q. Qualitative behavior of a discrete SIR epidemic model. Int. J. Biomath. 2016, 9, 1650092. [CrossRef]

50. GümüÅ̈̈, Ã.-z.A.; Selvam, A.G.; Vianny, D.A. Bifurcation and stability analysis of a discrete time SIR epidemic model with vaccination. Int. J. Anal. Appl. 2019, 17, 809-820.

51. Abdeljawad, T. On Riemann and Caputo fractional differences. Comput. Math. Appl. 2011, 62, 1602-1611. [CrossRef]

52. Wu, G.C.; Baleanu, D. Jacobian matrix algorithm for Lyapunov exponents of the discrete fractional maps. Commun. Nonlinear Sci. Numer. Simul. 2015, 22, 95-100. [CrossRef]

53. Petras, I. Fractional Order Chaotic Systems. MATLAB Central File Exchange. Available online: https:/ / www.mathworks.com/ matlabcentral/fileexchange/27336-fractional-order-chaotic-systems (accessed on 27 December 2021).

54. Gottwald, G.A.; Melbourne, I. The 0-1 test for chaos: A review. Chaos Detect. Predict. 2016, 915, $221-247$.

55. Ran, J. Discrete chaos in a novel two-dimensional fractional chaotic map. Adv. Differ. Equ. 2018, 2018, 1-2. [CrossRef]

56. Pincus, S.M. Approximate entropy as a measure of system complexity. Proc. Natl. Acad. Sci. USA 1991, 88, 2297-2301. [CrossRef] 